\title{
A Hopfield Neural Network for Channel Assignment Problem in Cellular Radio Networks
}

\author{
Omid Moradi \\ ACECR(jahad-e-daneshgahi), Isfahan University of technology \\ Isfahan, Iran \\ Tel: 98-91-3103-2136Ｅ-mail: moradi.acecr@gmail.com
}

\begin{abstract}
In wireless mobile communication system, radio spectrum is limited resource. However, efficient use of available channels has been shown to improve the system capacity. The role of a channel assignment scheme is to allocate channels to calls or mobiles in such a way as to minimize call blocking or call dropping probabilities, and also to maximize the quality of service. Channel assignment is known to be an NP-hard optimization problem. In this paper, a new channel-assignment algorithm using a modified Hopfield neural network is proposed. The channel-assignment problem is formulated as an energy-minimization problem that is implemented by a modified discrete Hopfield network. In this algorithm, an energy function is derived, and the appropriate interconnection weights between the neurons are specified. The interconnection weights between the neurons are designed in such a way that each neuron receives inhibitory support if the constraint conditions are violated and receives excitatory support if the constraint conditions are satisfied. The algorithm will be tested by solving seven benchmark problems, where the total number of frequencies varied from 73 to 533 . This new algorithm, together with the proposed regular interval initialization and new interconnection weights, has better performance results than the existing algorithms in all of the seven problems.
\end{abstract}

Keywords: Wireless communication, Channel assignment, Neural network

\section{Introduction}

Recently, the demands for cellular mobile communication systems have rapidly increased due to the portability and the availability of the systems. On the other hand, the electromagnetic frequency spectrum allocated for this purpose has been limited. As a result, the efficient use of the frequency spectrum or channels has gained the importance in order to meet the increasing demands. The channel assignment problem has been extensively studied to solve this important task in cellular mobile communication systems.

The channel assignment problem (CAP) in this paper is based on a common model. The service area of the system is divided into a large number of hexagonal cells. A cell composes a unit area to provide communication services, where every user is located in one cell. When a user requests a call for this system, a channel or frequency spectrum is assigned there to provide the communication service. This channel assignment must satisfy the constraints to avoid the radio interference between channels. Funabiki, Okutani and Nishikawa (2000) states that " Three types of constraints have usually been considered in CAP.

1) The Cochannel Constraint (CCC): The same channel cannot be reused in the cells within a certain distance from each other. A set of channel-reuse forbidden cells is called a cluster, where a different channel must be assigned to every call.

2) The Adjacent Channel Constraint (ACC): Adjacent channels cannot be assigned to adjacent cells simultaneously. In other words, any pair of channels in adjacent cells must have a specified distance. Note that the distance indicates the difference in the channel domain.

3) The Cosite Constraint (CSC): Any pair of channels in the same cell must have a specified distance. this distance for $C S C$ is usually larger than that for $A C C$.

The goal of CAP is to find a channel assignment to every requested call with the minimum number of channels subject to the above three constraints.' (p.397)

\subsection{Problem formulation of CAP}

Gamst and Rave(1982) defined the general form of the channel assignment problem in an arbitrary inhomogeneous cellular radio network. In their definition, the electromagnetic compatibility constraints in an $N$-cell network are described by an $N \times N$ symmetric matrix which is called compatibility matrix $C$. Each nondiagonal element $c_{i j}$ in $C$ represents the minimum separation distance in the frequency domain between a 
frequency assigned to cell $\# i$ and a frequency to cell $\# j$. The cochannel constraint $(C C C)$ is represented by $c_{i j}=$ 1 , and the adjacent channel constraint $(A C C)$ is represented by $c_{i j}=2 . c_{i j}=0$ indicates that cells $\# i$ and $\# j$ are allowed to use the same frequency. Each diagonal element $c_{i i}$ in $C$ represents the minimum separation distance between any two frequencies assigned to cell $\# i$, which is the cosite constraint $(C S C)$, where $c_{i j} \geq 1$ is always satisfied. The channel requirements for each cell in an $N$-cell network are described by an $N$-element vector which is called demand vector $D$. Each element $d_{i}$ in $D$ represents the required channel numbers(RCN's) to be assigned to cell $\# i$. When $f_{i k}$ indicates the $k$ th frequency assigned to cell $\# i$, the electromagnetic compatibility constraints are represented by:

$$
\begin{gathered}
\left|f_{i k}-f_{j l}\right| \geq c_{i j}, \text { for } i=1, \ldots, N, j=1, \ldots, N \\
k=1, \ldots, d_{i}
\end{gathered}
$$

and

$$
l=1, \ldots, d_{i}
$$

except

$$
i=j, k=l \text {. }
$$

The channel assignment problem in the cellular radio network is finding a conflict-free frequency assignment with the minimum number of total frequencies, where $C$ and $D$ are given(pp.309-315). Consider a channel assignment problem in a four-cell network proposed by He et al.(2002):

Fig. 1 shows the compatibility matrix $C$, The demand vector $D$, and the corresponding networks topology, as well as that of several interference-free optimum solutions with 11 channels. The network topology corresponds to the compatibility matrix $C$. The vertex represents a cell, and the edge represents the existence of $C C C$ or $A C C$ between two cells. The diagonal term $c_{i i}=5$ indicates that any two channels assigned to cell $\# i$ must be at least five channels apart in order to satisfy CSC. Channels assigned to cells \#1 and \#2 must be at least $c_{12}=4$ channels apart. Off-diagonal terms of $c_{i j}=1$ and $c_{i j}=2$ correspond to $C C C$ and $A C C$, respectively. The CAP, as demonstrated by using this example, tries to find a conflict-free channel assignment that satisfies the constraint conditions with the minimum number of total channels with given $C$ and $D$. Suppose that $M$ represents the number of channels available. Why is the minimum number of channels needed for an interference-free assignment 11 in this example? From Fig.1, because cell \#4 requires at least $11(=1+5 \times 2)$ channels, the minimum number of channels needed for an interference-free assignment in this example is 11 . Thus, $M=11$ is the lower bound, and we will be unable to find any interference-free assignments if $M<11$ (pp.1387-1388).

In this paper, an energy function is derived, which represents the constraints that should be satisfied in order to find the best assignment. The appropriate interconnection weights are designed such that the constraints on the channel-assignment problem are expressed in terms of inhibitory connections between neurons. A 2-D discrete Hopfield network is implemented to minimize the energy function. The state of each neuron in the Hopfield network represents the possibility of assigning a certain channel to a cell. To escape the local minima during the solution search, the assigned channel numbers (ACN's) in a cell are checked, and if the ACN's are less than the RCN's, then a forced frequency-assignment method is used. The forced frequency assignment method is an additional assignment of one or more channels although the energy is increased by the additional channel assignment since the extra assignment has violated the constraints. This simple technique dramatically increases the convergence rate of assignment since most of the local minima are due to a lack of assigned frequencies, which satisfy all three constraints for each cell. Initialization method and updating technique, which use the specific characteristics of the channel-assignment problem (e.g., CSC, CCC, and $A C C$ ) in cellular radio networks are investigated to reduce the number of iterations and to increase the convergence rate of channel assignment. The proposed frequency-assignment technique uses a modified discrete Hopfield network to achieve a solution that simultaneously satisfies all the constraints by using new interconnection weights and external inputs. In this paper channel assignment to the calls in a cell, which determines the lower bound on the total number of channels are fixed after regular interval initialization method.

Many researchers (Sivarajan, McEliece, and Ketchum, 1989; Gamast and Rave, 1982; Kunz, 1991; Funabiki and Takefuji, 1992; Chan, Palaniswami and Everitt, 1994; Kim et al., 1997; Funabiki, Okutani and Nishikawa, 2000; 
Vidyarthi, Ngom and Stojmenovic, 2005) have investigated the CAP in telephone networks. Sivarajan, McEliece and Ketchum (1989) proposed an $O(\mathrm{n} 2)$ time sequential heuristic algorithm, based on the first algorithm introduced by Gamst and Rave (1982). Sivarajan, McEliece and Ketchum (1989) applied their algorithm to several problems, where the values of total frequencies in solutions are shown without any actual assignment results(pp.846-850). Kunz (1991) used the continuous Hopfield network, where the output of each neuron $V_{i}$ was a fixed function $f$ of the internal state $u_{i}$, i.e., $V_{i}=f\left(u_{i}\right)$, where $f(x)=1 / 2(1+\tanh (\lambda x))$. The Kunz neural-network model required a large number of iterations in order to reach the final solution, and there were also difficulties in finding the proper values for and the parameters in the interconnection weights and energy function. Kunz (1991) considered cochannel and cosite interference in his neural-network model(pp.188-193). Funabiki and Takefuji (1992) proposed a neural-network parallel algorithm for channel-assignment problem. All input values are sequentially updated, while all output values are fixed. Then, all output values are sequentially updated, while all input values are fixed. Their neural-network model is composed of the hysteresis McCulloch-Pitts neurons. In the Funabiki and Takefuji model(1992), four heuristics were used to improve the convergence rate of channel assignment. They also fixed the channel assignment in one or more certain cells in order to accelerate the convergence time. The results were favorable in some cases, but not in others (PP.430-436). Chan, Palaniswami and Everitt (1994) used a feed forward neural network, which had a learning process prior to actual channel assignment. For the learning process, they used training data that was dependently obtained by other assignment methods. The performance of their algorithm is totally dependent on the used training data. Also, only the cochannel constraint (CCC) was considered (PP.279-288). Kim et al.(1997) used a modified Hopfild network. In their algorithm, used new interconnection weights an external inputs to the neurons with the initialization and updating methods(pp.957-967). Funabiki et al. (2000) proposed a three-stage algorithm of combining sequential heuristic methods into a parallel neural network for the NP-complete channel assignment problem(p.397). Vidyarthi et al.(2005) proposed a hybrid channel assignment approach using an efficient evolutionary strategy. They developed an evolutionary strategy(ES) which optimized the channel assignment(pp.1887-1895).

In this paper, a modified discrete Hopfield neural-network algorithm for the channel-assignment problem is proposed in order to improve the convergence rate and to reduce the number of iterations. My experimental results are also compared with that of Kim et al.(1997). In this paper, the channel-assignment problem is formulated as an energy-minimization problem such that the energy is at its minimum when all the constraints are satisfied and the number of assigned frequencies are the same as the required channel numbers (RCN's) in each cell.

\section{Modified Hopfield Network for Channel Assignment}

Each processing element (neuron) is fully interconnected in the Hopfield network. The $i$ th neuron is described by its state, which is denoted by $V_{i}$. Each neuron has two possible states. The value of each state is determined by the total input from other neurons followed by a thresholding operation. The input of the $i$ th neuron is derived from two sources: the outputs of other neurons scaled by the connection weights and an appropriate external input. The total input to neuron $\# i$ is denoted by $U_{i}$

$$
U_{i}=\sum_{j} W_{i j} V_{j}+I_{i}
$$

where $W_{i j}$ is the connection weight from neuron $\# j$ to neuron $\# i$ and $I_{i}$ is the external input. Each neuron updates its own state according to a thresholding rule with threshold $T H D$ as shown by

$$
V_{i}=\left\{\begin{array}{rr}
1 & \text { if } U_{i}>T H D \\
0 & \text { otherwise }
\end{array}\right.
$$

The thresholding rule can be applied asynchronously (in series) or synchronously (in parallel). In the asynchronous mode, this rule is applied sequentially to each neuron, and the state of each neuron is updated individually. In the synchronous mode, this thresholding operation is simultaneously applied to every neuron, and the states of all neurons are updated at the same time. The updating operation is terminated when the states are unchanged or the energy has reached a minimum value. According to Kim et al.(1997), The energy function $E$ is defined as

$$
E=-\frac{1}{2} \sum_{i} \sum_{j} W_{i j} V_{i} V_{j}-\sum_{i} V_{i} I_{i}
$$

This energy function is minimized by the Hopfield neural network updating procedure. The successive application of the updating procedure will force the network to converge such that the energy of the network 
becomes smaller during the updating procedure. When the network reaches a stable state, it has fallen into minimum energy state, where this could be a local or global minimum. $W_{i j}$ and $I_{i}$ should be set appropriately for the applications so that $E$ represents the function, which is minimized to solve the combinatorial optimization problems. The energy function should represent all the constraints of the problem.

In my algorithm, a discrete Hopfield network is implemented, which is updated until the energy is equal to zero or a predefined maximum iteration number has been reached. In the simulation, I considered a mobile radio network that has $N$ cells (or base stations) and $M$ available frequency channels. The value of $M$ is set to be the lower bound of required number of channels $L B$ for a given channel assignment problem. Fig.2 shows a two-dimensional (2-D) Hopfield network for the channel-assignment problem. Each $i$ th cell can carry any of the $d_{i}$ frequencies among the $M$ frequencies if the carriage of frequencies does not violate the imposed constraints of the channel-assignment problem. The value of the processing unit $V_{i j}, 1 \leq i \leq N$ and $1 \leq j \leq M$ indicates if frequency $\# j$ is assigned to cell $\# i$ : $V_{i j}=1$ meaning that the frequency $j$ is assigned to cell $\# i$ and $V_{i j}=0$, which means that the frequency $\# j$ cannot be assigned to cell $\# i$. In Fig.2, $i$ and $p$ represent the cell number; $j$ and $q$ indicate the frequency number, respectively. The state of the current processing neuron to be updated is represented by $V_{i j}$. An energy function is derived to represent the three constraints of the channel-assignment problem. In $C S C$, a frequency $f_{i q}$ cannot be assigned to cell $\# i$ if the distance of $f_{i q}$ and any assigned frequency $f_{i j}$, $1 \leq j \leq M$ is less than $c_{i i}$ (minimum frequency distance for CSC). The energy function for cell \#i (for CSC) can be defined as

$$
E_{C S C}^{i}=\sum_{j=1}^{M} \sum_{q=1}^{M} V_{i j} V_{i q} X_{i j q}
$$

where

$$
X_{i j q}= \begin{cases}1, & \text { if } q \neq j \quad \text { and } \\ & j-\left(c_{i i}-1\right) \leq q \leq j+\left(c_{i i}-1\right) \\ 0, & \text { otherwise. }\end{cases}
$$

For both $A C C$ and $C C C$, the frequency $f_{i j}$ cannot be assigned to cell $\# i$ if the distance of $f_{i j}$ and any other assigned frequency $f_{p q}$ is less than $c_{i p}$ (minimum frequency separation between the frequencies in cell $\# i$ and cell $\# p$ ). The energy function of $i$ th cell for $A C C$ and $C C C$ can be defined as

$$
E_{(A C C, C C C)}^{i}=\sum_{j=1}^{M} \sum_{p=1}^{N} \sum_{q=1}^{M} V_{i j} V_{p q} Y_{i j p q}
$$

Where

$$
Y_{i j p q}= \begin{cases}1, & \text { if } p \neq i \text { and } \\ & c_{i p}>0 \text { and } j-\left(c_{i p}-1\right) \leq q \leq j+\left(c_{i p}-1\right) \\ 0, & \text { otherwise. }\end{cases}
$$

In addition to the three constraint conditions, the total ACN's in the $i$ th cell must be the same as the RCN's for cell $\# i$. The energy function for the ACN can be defined as

$$
E_{A C N}^{i}=\left(d_{i}-\sum_{j=1}^{M} V_{i j}\right)^{2}
$$

From (5), (7), and (9), the energy function for $i$ th cell can be defined as

$$
E^{i}=\left(d_{i}-\sum_{j=1}^{M} V_{i j}\right)^{2}+\sum_{j=1}^{M} \sum_{q=1}^{M} V_{i j} V_{i q} X_{i j q}+\sum_{j=1}^{M} \sum_{p=1}^{N} \sum_{q=1}^{M} V_{i j} V_{p q} Y_{i j p q}
$$

The total energy function for the channel-assignment problem is as follows:

$$
E=\sum_{i=1}^{N}\left(\left(d_{i}-\sum_{j=1}^{M} V_{i j}\right)^{2}+\sum_{j=1}^{M} \sum_{q=1}^{M} V_{i j} V_{i q} X_{i j q}+\sum_{j=1}^{M} \sum_{p=1}^{N} \sum_{q=1}^{M} V_{i j} V_{p q} Y_{i j p q}\right)
$$


The channel-assignment problem is now formulated as an energy-minimization problem. This energy function can be minimized by an equivalent Hopfield neural network with the appropriate interconnection weights and the external inputs. The interconnection weights must represent the constraints of the optimization problem such as the RCN's for each cell and the three constraint of the channel-assignment problem. Each of the constraints are invoked by inhibitory and excitatory support. If neuron $V_{i j}$ takes a value of unity, which means frequency $f_{i j}$ can be used at cell $\# i$, then the neuron $V_{i q}$ within the interference must be inhibited by the CSC condition. The constraint can be specified in the form

$W_{C S C}=-\delta_{i p} \alpha_{j q}\left(c_{i i}\right)$

where $\delta$ is the Kronecker delta function and defined with $\alpha_{i j}$ as follows:

$\delta_{i j}= \begin{cases}1 . & \text { if } i=j \\ 0 & \text { otherwise }\end{cases}$

$\alpha_{i j}(x)= \begin{cases}1, & \text { if }|i-j|<x \\ 0, & \text { otherwise. }\end{cases}$

In both $A C C$ and $C C C$, if neuron $V_{i j}$ takes a value of unity, then a neuron $V_{i q}$ within the interference must be inhibited. This constraint can be specified in the form

$$
W_{(A C C, C C C)}=-\left|\left(1-\delta_{i p}\right)\right| \alpha_{j q}\left(c_{i j}\right)
$$

To make ACN's the same as the RCN's, I have to feed inhibitory support to the neuron by an amount proportional to the number of assigned frequencies. If RCN is less than or equal to the ACN's, the additional frequencies cannot be assigned to a cell. This constraint can be expressed as

$$
W_{A C N}=-\delta_{i p}\left|\left(1-\delta_{j q}\right)\right|
$$

Equation (16) shows that the self-inhibition is not allowed. The purpose of $W_{A C N}$ is to give the inhibitory support to neurons in the same cell in order to avoid the case of ACN $>$ RCN.

From (12), (15), and (16), the total interconnection weight is

$$
W_{i j p q}=-\delta_{i p} \alpha_{j q}\left(c_{i i}\right)-\left|\left(1-\delta_{i p}\right)\right| \alpha_{j q}\left(c_{i j}\right)-\delta_{i p}\left|\left(1-\delta_{j q}\right)\right|
$$

The interconnection weight $W_{i j p q}$ between $V_{i j}$ and $V_{p q}$ is symmetrical, i.e., $W_{j p q}=W_{p q i j}$ for $1 \leq i, p \leq N$ and $1 \leq j$, $q \leq M$. Self-feedback is not allowed, i.e., $W_{i j i j}=0$.

In this paper, to increase the convergence rate, a nonlinear function is applied on the weights as follows

$$
W_{i j p q(\text { new })}=A \times \operatorname{sign}\left(W_{i j p q}\right) \times \exp \left(\left|W_{i j p q}\right|\right)
$$

The external input $I_{i}$ is defined as

$I_{i}=\left(d_{i}-1\right)$

The external input $I_{i}$ is used to give excitatory support to neurons in the same cell to make them to be satisfied by the traffic demand constraint. If a frequency assignment satisfies all the three constraints $(C S C, C C C$, and $A C C$ ) for a cell and the ACN's are less than the RCN's, then that cell must receive excitatory support as it relates to reinforcing that assignment. The summation of inputs from all neurons for the current updating neuron is $-\left(d_{i}\right.$ -1) which should be given an excitatory bias. The input to each neuron $(i, j)$ in the original Hopfield neural net is defined as

$$
U_{i j}=\sum_{p=1}^{N} \sum_{q=1}^{M} W_{i j p q} V_{p q}+I_{i}
$$

When a neuron receives an input, only frequencies which are satisfied by the three channel-assignment constraints are selected as usable frequencies for the cell. A local minimum can be achieved for the channel assignment given in (20) by using the Hopfield updating procedure. If all the assigned frequencies are satisfied by the three channel-assignment constraints, but one or more of the cells have fewer channels than the RCN's, the frequency assignment will never change and the energy value cannot reach the global minima even though more iterations are performed. To prevent this from occurring, I incorporate a forced assignment method, which allows for a frequency to be assigned to a cell by another excitatory input, even though the channel-assignment 
constraints are violated and the energy is increased. The forced assigned frequency can change the frequency assignment for other cells too. The algorithm will then search for another solution space in order to attempt to reach the global minima when the current assigned channels does not satisfied the traffic demand constraint. The algorithm checks for the ACN with the value of RCN. The difference between the RCN and the ACN constraints is used as an additional excitatory input, which is given by

$$
I_{E_{i j}}=\left(d_{i}-\sum_{q=1}^{M} V_{i q}\right)
$$

The values of $W_{A C N}$ and $I_{i}$ are constant for the neurons in the same cell. However, the value of $I_{E i j}$ is variable based on the current neuron states. In $I_{E i j}$, the difference of (RCN-CAN) is fed into the neurons in cell \#i. To count $\mathrm{ACN}, V_{i j}$ is included in $\mathrm{ACN}$ of (21).

It is possible that the forced reassignment fails, i.e., $\mathrm{ACN}<\mathrm{RCN}$ although the additional term $I_{E i j}$ is introduced. If $\mathrm{ACN}<\mathrm{RCN}$ even after the forced reassignment is applied, it means that the assignment for some calls are failed, which will cause the call drops. The number of callers who could not have the channels and their calls are dropped is (RCN-ACN). This case is considered as a nonconvergence case. On the other hand, when all calls have the assigned frequencies without any call dropping, it is considered as convergence case. In the simulation results, the convergence rate is the number of cases that all calls have the assigned frequencies without any call dropping before the maximum number of iterations is reached when 100 simulation runs were performed. Input to each neuron of the modified network is defined as

$U_{i j}=\sum_{p=1}^{N} \sum_{q=1}^{M} W_{i j p q} V_{p q}+I_{i}+I_{E i j}$

The schematic description of the input to a neuron is shown in Fig.3. The output function consists of a threshold operation. The final output state of the neuron is

$V_{i j}=f_{\text {out }}\left(U_{i j}\right)$

Where

$f_{\text {out }}= \begin{cases}1, & \text { if } U_{i j} \geq T H D \\ 0, & \text { otherwise }\end{cases}$

and $T H D$ is the threshold value and $T H D=0$ in this paper.

\section{Applying Modified Hopfield Network}

In this section, the implementation of the algorithm is discussed. The overall algorithm is summarized in the following steps.

1) The initial state of neurons is set to one or zero according to the initialization method.

2) Repeat the following steps until all neurons are picked.

a) Pick neuron $V_{i j}$ according to the updating method.

b) Calculate the input to this neuron by (22).

c) Decide the new state of this neuron by (23) and (24).

3) Compute the energy $E$ of the current assignment. If $E=0$, stop and go to Step 4), otherwise, repeat the process from Step 2).

4) The output state of the neurons $V_{i j}$ will be the final assignment based on the compatibility matrix $C$ and demand vector $D$.

\subsection{Initialization method}

In general, a random initialization method is used as the initial states for the neurons in the Hopfield network. In my algorithm, initialization technique with the frequency assignment constraints are investigated in order to increase the convergence rate and to decrease the iteration number. The total frequency spectrum is composed of a certain number of blocks for the initialization with consideration of the constraints. Fig.4(a) shows the frequency block structures. $M$ is the total number of frequencies, and the total frequency spectrum consists of $B$ blocks. Every frequency block except the last one has the same number of frequency slots. The last block might not have the same number of frequency slots depending on the $L B$ and the block width $(B W)$, which is the 
number of channels in the block. In Fig.4(a), for example, all blocks have $B W_{i}=5,1 \leq i \leq(B-1)$ except the last block $B$, which has $\mathrm{BW}_{\mathrm{B}}=2$. Frequency $j$ refers to the number of each frequency slot, with each slot identified by $f_{i j}, 1 \leq i \leq N, 1 \leq j \leq M$. The channel number in block (CNB) is the frequency number in the block, and the sequence is repeated for each block.

In my algorithm, the initial state of neurons, $V_{i j}=0$ or $V_{i j}=1$, where $1 \leq i \leq N, 1 \leq j \leq M$ indicates whether or not the frequency slot $f_{i j}$ is to be assigned to the $j$ th call in the cell $i$. Fig. 4(b) shows the fixed interval initialization method, which will be explained later in detail. In Fig. 4(b), the frequency slots with the initial state value $V_{i j}=1$ are indicated by the dark slots. In the frequency-assignment network, the lower bound $(L B)$ on the total of channels can be obtained from demand vector $D$ and the compatibility matrix $C$ for the cell $C_{d m a x}$. The distance between the frequencies for a certain cell should be at least $c_{i i}$. $L B$ for the network is defined by

$$
L B=\left(d_{\max }-1\right) \cdot c_{i i}+1
$$

The $B W$ is defined by

$$
B W= \begin{cases}c_{i i} & \text { if } M=L B \\ \left.\frac{M}{d_{\max }-1}\right\rfloor & \text { otherwise }\end{cases}
$$

where $\lfloor a\rfloor$ is the largest integer, which is equal or less than $a$. The number of blocks $B$ is defined by

$$
B=\left\lceil\frac{M}{B W}\right\rceil
$$

Where $\lfloor a\rfloor$ is the smallest integer, which is equal or greater than $a$. The procedure of regular interval initialization method is as follows.

1) For the cell $\# i$ with $d_{i}$ calls, randomly choose the frequency block and channel number in the chosen block (CNB) $\mathrm{k}, 1 \leq k \leq B W$ in the chosen frequency block, which does not violate $C C C$ and $A C C$ with the previously assigned frequencies of other cells. Assign a "one" to the chosen frequency slot, $V_{i k}=1$. If the cell $\# i$ is $C_{d \max }, k=1$.

2) For the remaining calls of the cell, assign a "one"to the frequency slot, which has the distance BW with the previous assigned slots, $V_{i k^{\prime}}=1$, where $k^{\prime}=\left(k+d_{f} . B W\right) \bmod M$ for $d_{f}=1,2, \ldots, d_{i}-1$.

3) Fixed channel assignment for the cell $C_{d m a x}$.

\subsection{Updating Method}

In an asynchronous Hopfield network, the neurons are selected randomly or sequentially by a certain order for the updating. In my algorithm, both random and sequential selection techniques are used along with updating method.

According to Kim et al.(1997), The procedural step for updating method is as follows.

1) Make a list of cells according to the descending order of RCN for each cell.

2) Execute the iteration subroutine as follows until the counter reached to a prespecified maximum iteration number 500 or $E=0$.

a) Choose the cell $\# i$ according to the order of the cell list.

b) Randomly choose one neuron $\# j$ in cell $\# i[$ neuron $(i, j)]$ and update that neuron.

c) For the next updating neuron, the direction is randomly chosen whether in favor of the left- [neuron $(i, j-1)$ ] or the right-side neuron [neuron $(i, j+1)]$.

d) After the initial direction is decided by Step $c$ ), the next neurons are updated sequentially.

e) Repeat Steps a) - d) until all frequencies for all the cells are assigned.

3) Repeat Step 2) for the next cell in the list (pp.963-964).

\section{Simulation and Discussion}

A mobile system consisting of 21 or 25 cells is used in my simulation. The 21-cell system is repeated in Fig. 5 . The output threshold (THD) in (24) is fixed at $\mathrm{THD}=0$. The simulation results according to the regular interval 
initialization method and new interconnection weight will be discussed and then compared with that of Kim et al.(1997) in this paper.

Table I shows the specifications of the problems, which are also used by Kim et al.(1997). $L B$ is the lower bound on required frequencies for each problem. Figs.6 shows the compatibility matrices and the demand vectors for these problems. In Table II, the result of this paper with new interconnection weight and initialization method are compared with the result in Kim et al.'s (1997) algorithm. The average iteration number and the convergence rate to the solution are also shown in Table II. The average iteration number is the average number of iterations, which are increased until $E=0$. Convergence rate is the probability that the network has $E=0$ before the maximum number iteration is reached. In these simulations, the maximum number of iterations is fixed at 500 . To investigate the number of iterations and the convergence rates, 100 simulation runs were performed with different initial seed values using a random number generator for each of the seven problems. In table II, the simulation results in this paper have a smaller average iteration number and a higher convergence rate than Kim et al.'s (1997) results. For example, in problem \#1, my algorithm found the solution with 156.23 average iteration number and 95\% convergence rate, but Kim et al.'s (1997) algorithm found the solution with 263.4 average iteration number and $61 \%$ convergence rate. It demonstrates that generally, my algorithm has a better performance (i.e., smaller iteration numbers and higher convergence rates) than Kim et al.'s (1997) algorithm. It also shows that my proposed initialization method with fixed channel assignment for the cell $C_{d m a x}$, has better performance than the random initialization method which is usually used. For example, fig. 7 and Table III shows the distribution of the frequency for each cell in problem \#1 and cell \#9 has the largest element in the demand vector, so the frequency assignment of cell \#9 was fixed in this algorithm. Fixing a single cell frequency assignment can drastically reduce the searching space and consequently the convergence time is shortened. In addition, Fig.8 shows two typical trajectory of problem \#1 and \#2 that demonstrates the convergence rate.

\section{Conclusion}

In this paper, a modified Hopfield neural-network algorithm is proposed in order to obtain an optimal solution for the channel-assignment problem in the mobile cellular environment. This improved algorithm drives itself to the solution by using new interconnection weights and external inputs to the neurons with the initialization and updating method. The interconnection weight is set initially to take into account the RCN in each cell and the three channel-assignment constraints. The three constraints that are considered are the $C C C, A C C$, and $C S C$. The compatibility matrix $C$, demand vector $D$, and the lower bound number of total frequencies are presented as input. In the simulation results, the average iteration number and convergence rates are shown and compared with that of Kim et al.'s(1997) results. The proposed algorithm using the suggested initialization method and new interconnection weight has a better performance than Kim et al.'s(1997) algorithm. This algorithm is simple to implement in very large scale integration (VLSI) since the algorithm has only simple functions such as adder, inverter, and comparator. Also, it has a modular structure since each neuron can be constructed as a module.

\section{References}

Chan, P. T. H., Palaniswami, M. \& Everitt, D. (1994). Neural network based dynamic channel assignment for cellular mobile communication systems, IEEE Trans. Veh. Technol., vol. 43, pp. 279-288.

Funabiki, N., \& Takefuji, Y. (1992). A neural network parallel algorithm for channel assignment problems in cellular radio networks. IEEE Trans. Veh. Technol., vol.41,pp. 430-436.

Funabiki, N., Okutani, N. \& Nishikawa, S. (2000). A Three-Stage Heuristic Combined Neura -Network Algorithm for Channel Assignment in Cellular Mobile Systems. IEEE Trans. Veh. Technol., vol.49,pp. $397-403$.

Gamst, A. \& Rave, W. (1982). On frequency assignment in mobile automatic telephone systems, in roc. GLOBECOM, pp. 309-315.

He, Z., Zhang, Y., Wei, C. \& Wang, J. (2002). A multistage self-organizing algorithm combined transiently chaotic neural network for cellular channel assignment, IEEE Trans. Veh. Technol., vol.51,NO.4, pp. 1386-1396.

Kunz, D. (1991). Channel assignment for cellular radio using neural networks. IEEE Trans. Veh. Technol., Vol.40,pp. 188-193.

Kim, J. S., Park, S. H., Dowd, P. W. \& Nasrabadi, N. M. (1997). Cellular radio channel assignment using a modified hopfield network. IEEE Trans. Veh. Technol.,vol.46,pp. 957-967.

Moradi, O. (2010). Fixed channel assignment and neural network algorithm for channel assignment problem in cellular radio networks. Canadian CIS Journal, vol.3,pp.93-103.

Sivarajan, K. N. , McEliece, R. J., \& Ketchum, J. W. (1989). Channel assignment in cellular radio. IEEE Vehicle 
Technology Society Conference. pp. 846-850.

Vidyarthi, G., Ngom, A. \& Stojmenovic, I. (2005). A hybrid channel assignment approach using an efficient evolutionary strategy in wireless mobile networks, IEEE Trans. Veh. Technol., vol.54,PP.1887-1895.

Table 1. Specification of Simulation Problems.

\begin{tabular}{ccccc}
\hline $\begin{array}{c}\text { Problem } \\
\#\end{array}$ & $\begin{array}{c}\text { Number of } \\
\text { Radio Cells }\end{array}$ & $\begin{array}{c}\text { Lower } \\
\text { bound } \\
\text { L }\end{array}$ & $\begin{array}{c}\text { Compatibility } \\
\text { Matrix } \\
C\end{array}$ & $\begin{array}{c}\text { Demand } \\
\text { Vector } \\
D\end{array}$ \\
\hline 1 & 25 & 73 & $C_{2}$ & $D_{2}$ \\
2 & 21 & 381 & $C_{3}$ & $D_{3}$ \\
3 & 21 & 533 & $C_{4}$ & $D_{3}$ \\
4 & 21 & 533 & $C_{5}$ & $D_{3}$ \\
5 & 21 & 221 & $C_{3}$ & $D_{4}$ \\
6 & 21 & 309 & $C_{4}$ & $D_{4}$ \\
7 & 21 & 309 & $C_{5}$ & $D_{4}$ \\
\hline
\end{tabular}

Table 2. Summary of Simulation Results

\begin{tabular}{|c|c|c|c|c|c|c|}
\hline \multirow{2}{*}{ Problem \# } & \multirow{2}{*}{$L B$} & \multirow{2}{*}{$M$} & \multicolumn{2}{|c|}{ Hopfield network } & \multicolumn{2}{c|}{ Kim's et al. } \\
\cline { 4 - 7 } & & & $\begin{array}{c}\text { Average Iter. } \\
\text { No. }\end{array}$ & $\begin{array}{c}\text { Convergence } \\
\text { Rate }\end{array}$ & $\begin{array}{c}\text { Average Iter. } \\
\text { No. }\end{array}$ & $\begin{array}{c}\text { Convergence } \\
\text { Rate }\end{array}$ \\
\hline 1 & 73 & 73 & 156.23 & $95 \%$ & 263.4 & $61 \%$ \\
2 & 381 & 381 & 11.59 & $100 \%$ & 67.1 & $100 \%$ \\
3 & 533 & 533 & 3.34 & $100 \%$ & 85.9 & $98 \%$ \\
4 & 533 & 533 & 44.78 & $100 \%$ & 129.5 & $99 \%$ \\
5 & 221 & 221 & 28.59 & $98 \%$ & 65.6 & $95 \%$ \\
6 & 309 & 309 & 32.44 & $100 \%$ & 118.2 & $96 \%$ \\
7 & 309 & 309 & 64.77 & $60 \%$ & 119.1 & $40 \%$ \\
\hline
\end{tabular}


Table 3. Distribution of the frequency for each cell with 533 frequency in problem \#3.

\section{Cell number}

\begin{tabular}{|c|c|c|c|c|c|c|c|c|c|c|c|c|c|c|c|c|c|c|c|c|}
\hline 1 & 2 & 3 & 4 & 5 & 6 & 7 & 8 & 9 & 10 & 11 & 12 & 13 & 14 & 15 & 16 & 17 & 18 & 19 & 20 & 21 \\
\hline 16 & 17 & 188 & 24 & 33 & 43 & 18 & 3 & 1 & 2 & 20 & 5 & 5 & 1 & 4 & 5 & 7 & 17 & 16 & 6 & 48 \\
\hline 61 & 25 & 201 & 167 & 89 & 97 & 34 & 11 & 8 & 10 & 30 & 50 & 31 & $\begin{array}{c}1 \\
29\end{array}$ & & 13 & 21 & 151 & 51 & 14 & $\begin{array}{l}40 \\
76\end{array}$ \\
\hline 124 & 40 & 215 & 196 & 189 & 137 & 45 & 20 & 15 & 28 & 46 & 92 & 58 & 104 & 19 & 23 & 31 & 173 & 126 & 130 & 124 \\
\hline 212 & 94 & 275 & 227 & 197 & 244 & 93 & 27 & 22 & 42 & 84 & 99 & 88 & 113 & 28 & 32 & 38 & 327 & 277 & 150 & 332 \\
\hline 457 & 149 & 322 & 346 & 235 & 260 & 100 & 35 & 29 & 52 & 214 & 117 & 118 & 162 & 53 & 46 & 58 & 348 & 304 & 198 & 369 \\
\hline 487 & 185 & 329 & 499 & 306 & 275 & 140 & 47 & 36 & 86 & 299 & 138 & 126 & 180 & 63 & 59 & 74 & 410 & 364 & 231 & 392 \\
\hline 511 & 192 & 455 & 513 & 375 & 295 & 150 & 69 & 43 & 93 & 326 & 148 & 159 & 201 & $\begin{array}{l}70 \\
83\end{array}$ & 72 & 103 & 454 & 462 & 282 & 404 \\
\hline 525 & 200 & 485 & 530 & 497 & 330 & 255 & 82 & 50 & 123 & 341 & 176 & 168 & 242 & $\begin{array}{c}83 \\
107\end{array}$ & 81 & 112 & 504 & 482 & 349 & 499 \\
\hline & 209 & & & & 352 & 300 & 91 & 57 & 137 & 364 & 201 & 176 & 269 & 102 & 88 & 122 & & 517 & 377 & \\
\hline & 221 & & & & 379 & 322 & 101 & 64 & 144 & 371 & 222 & 202 & 282 & $\begin{array}{l}125 \\
142\end{array}$ & 97 & 199 & & 529 & 395 & \\
\hline & 242 & & & & 431 & 378 & 108 & 71 & 168 & 408 & 264 & 209 & 327 & 142 & 107 & 212 & & & 406 & \\
\hline & 276 & & & & 482 & 385 & 118 & 78 & 194 & 416 & 278 & 223 & 411 & 151 & 117 & 257 & & & 515 & \\
\hline & 294 & & & & 499 & 395 & 132 & 85 & 208 & 508 & 405 & 230 & 486 & $\begin{array}{l}104 \\
181\end{array}$ & 128 & 301 & & & 523 & \\
\hline & 339 & & & & 526 & 404 & 139 & 92 & 237 & & 415 & 253 & 496 & 181 & 136 & 334 & & & & \\
\hline & 349 & & & & 533 & 412 & 146 & 99 & 248 & & 458 & 262 & 509 & 2016 & 143 & 345 & & & & \\
\hline & 364 & & & & & 493 & 167 & 106 & 258 & & & 271 & & 210 & 154 & 352 & & & & \\
\hline & 375 & & & & & 503 & 177 & 113 & 272 & & & 291 & & 224 & 161 & 360 & & & & \\
\hline & 392 & & & & & 530 & 184 & 120 & 283 & & & 312 & & $\begin{array}{l}230 \\
247\end{array}$ & 175 & 367 & & & & \\
\hline & 422 & & & & & & 193 & 127 & 318 & & & 323 & & & 186 & 387 & & & & \\
\hline & 459 & & & & & & 206 & 134 & 328 & & & 342 & & 201 & 196 & 403 & & & & \\
\hline & 469 & & & & & & 214 & 141 & 342 & & & 351 & & $\begin{array}{l}272 \\
287\end{array}$ & 203 & 413 & & & & \\
\hline & 482 & & & & & & 226 & 148 & 366 & & & 358 & & 201 & 210 & 423 & & & & \\
\hline & 496 & & & & & & 238 & 155 & 390 & & & 386 & & $\begin{array}{l}311 \\
328\end{array}$ & 217 & 437 & & & & \\
\hline & 504 & & & & & & 245 & 162 & 439 & & & 409 & & 328 & 227 & 447 & & & & \\
\hline & 515 & & & & & & 256 & 169 & 448 & & & 417 & & $\begin{array}{l}335 \\
346\end{array}$ & 234 & 460 & & & & \\
\hline & & & & & & & 263 & 176 & 457 & & & 440 & & $\begin{array}{l}346 \\
354\end{array}$ & 241 & 478 & & & & \\
\hline & & & & & & & 271 & 183 & 468 & & & 472 & & $\begin{array}{l}354 \\
373\end{array}$ & 251 & 486 & & & & \\
\hline & & & & & & & 279 & 190 & 503 & & & 490 & & $\begin{array}{l}373 \\
410\end{array}$ & 259 & 506 & & & & \\
\hline & & & & & & & 290 & 197 & & & & 497 & & $\begin{array}{l}410 \\
418\end{array}$ & 268 & & & & & \\
\hline & & & & & & & 298 & 204 & & & & 506 & & 418 & 280 & & & & & \\
\hline & & & & & & & 305 & 211 & & & & 521 & & 427 & 293 & & & & & \\
\hline & & & & & & & 314 & 218 & & & & & & $\begin{array}{r}434 \\
\end{array}$ & 303 & & & & & \\
\hline & & & & & & & 332 & 225 & & & & & & $\begin{array}{l}441 \\
480\end{array}$ & 312 & & & & & \\
\hline & & & & & & & 350 & 232 & & & & & & $\begin{array}{l}480 \\
488\end{array}$ & 321 & & & & & \\
\hline & & & & & & & 357 & 239 & & & & & & $\begin{array}{l}488 \\
510\end{array}$ & 333 & & & & & \\
\hline & & & & & & & 369 & 246 & & & & & & $\begin{array}{l}510 \\
524\end{array}$ & 340 & & & & & \\
\hline & & & & & & & 380 & 253 & & & & & & & 347 & & & & & \\
\hline & & & & & & & 388 & 260 & & & & & & & 355 & & & & & \\
\hline & & & & & & & 397 & 267 & & & & & & & 362 & & & & & \\
\hline & & & & & & & 405 & 274 & & & & & & & 374 & & & & & \\
\hline & & & & & & & 417 & 281 & & & & & & & 381 & & & & & \\
\hline & & & & & & & 425 & 288 & & & & & & & 389 & & & & & \\
\hline & & & & & & & 443 & 295 & & & & & & & 396 & & & & & \\
\hline & & & & & & & 454 & 302 & & & & & & & 409 & & & & & \\
\hline & & & & & & & 465 & 309 & & & & & & & 416 & & & & & \\
\hline & & & & & & & 472 & 316 & & & & & & & 429 & & & & & \\
\hline & & & & & & & 481 & 323 & & & & & & & 438 & & & & & \\
\hline & & & & & & & 490 & 330 & & & & & & & 446 & & & & & \\
\hline & & & & & & & 500 & 337 & & & & & & & 453 & & & & & \\
\hline & & & & & & & 507 & 344 & & & & & & & 467 & & & & & \\
\hline & & & & & & & 516 & 351 & & & & & & & 474 & & & & & \\
\hline & & & & & & & 532 & 358 & & & & & & & 483 & & & & & \\
\hline & & & & & & & & 365 & & & & & & & 492 & & & & & \\
\hline & & & & & & & & 372 & & & & & & & 502 & & & & & \\
\hline & & & & & & & & 379 & & & & & & & 513 & & & & & \\
\hline & & & & & & & & 386 & & & & & & & 520 & & & & & \\
\hline & & & & & & & & 393 & & & & & & & 528 & & & & & \\
\hline & & & & & & & & 400 & & & & & & & & & & & & \\
\hline & & & & & & & & 407 & & & & & & & & & & & & \\
\hline & & & & & & & & 414 & & & & & & & & & & & & \\
\hline & & & & & & & & 421 & & & & & & & & & & & & \\
\hline & & & & & & & & 428 & & & & & & & & & & & & \\
\hline & & & & & & & & 435 & & & & & & & & & & & & \\
\hline & & & & & & & & 442 & & & & & & & & & & & & \\
\hline & & & & & & & & 449 & & & & & & & & & & & & \\
\hline & & & & & & & & 456 & & & & & & & & & & & & \\
\hline & & & & & & & & 463 & & & & & & & & & & & & \\
\hline & & & & & & & & 470 & & & & & & & & & & & & \\
\hline & & & & & & & & 477 & & & & & & & & & & & & \\
\hline & & & & & & & & 484 & & & & & & & & & & & & \\
\hline & & & & & & & & 491 & & & & & & & & & & & & \\
\hline & & & & & & & & 498 & & & & & & & & & & & & \\
\hline & & & & & & & & 502 & & & & & & & & & & & & \\
\hline & & & & & & & & 512 & & & & & & & & & & & & \\
\hline & & & & & & & & 519 & & & & & & & & & & & & \\
\hline & & & & & & & & 526 & & & & & & & & & & & & \\
\hline & & & & & & & & 533 & & & & & & & & & & & & \\
\hline
\end{tabular}




$$
C=\left[\begin{array}{llll}
5 & 4 & 0 & 0 \\
4 & 5 & 0 & 1 \\
0 & 0 & 5 & 2 \\
0 & 1 & 2 & 5
\end{array}\right]
$$$$
D=\left[\begin{array}{l}
1 \\
1 \\
1 \\
3
\end{array}\right]
$$

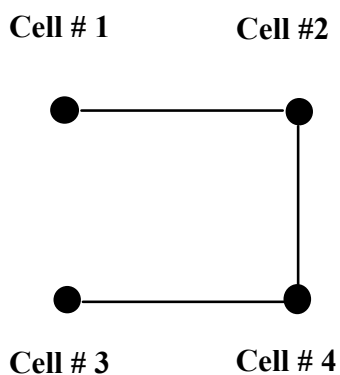

$\mathrm{V}_{\mathrm{ij}} \quad$ Frequency $\# j$

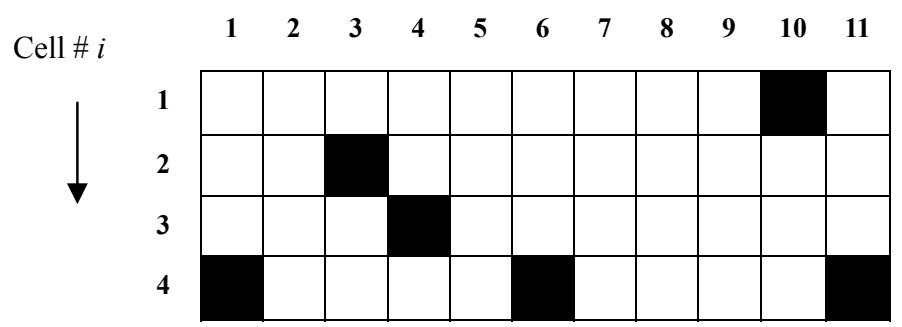

Figure 1. A CAP: compatibility matrix $C$, required $D$, the corresponding network topology, and the optimum solution with 11 channels

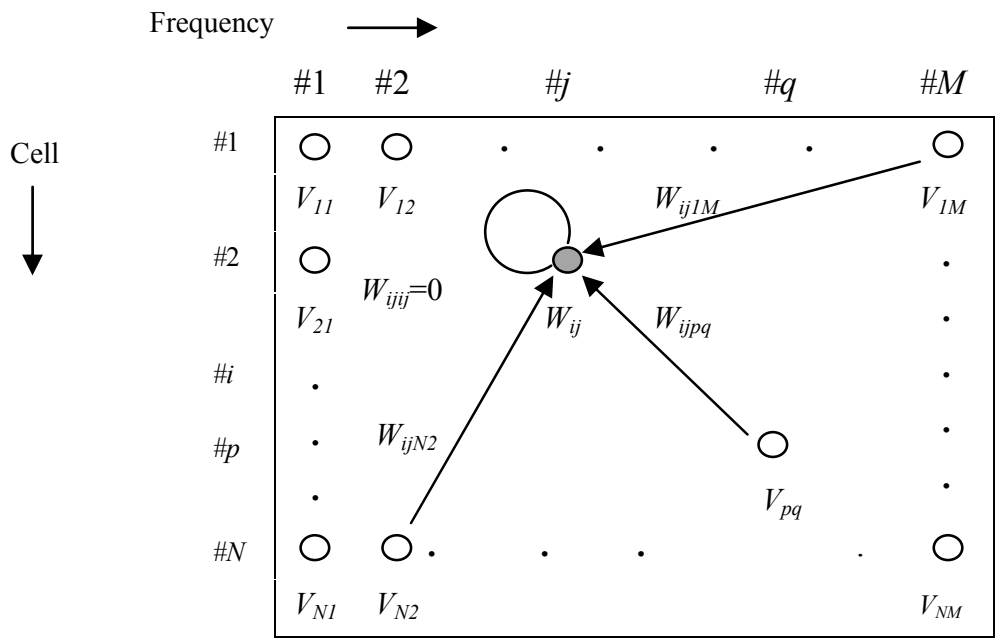

Figure 2. A 2-D Hopfield network for the channel-assignment problem 


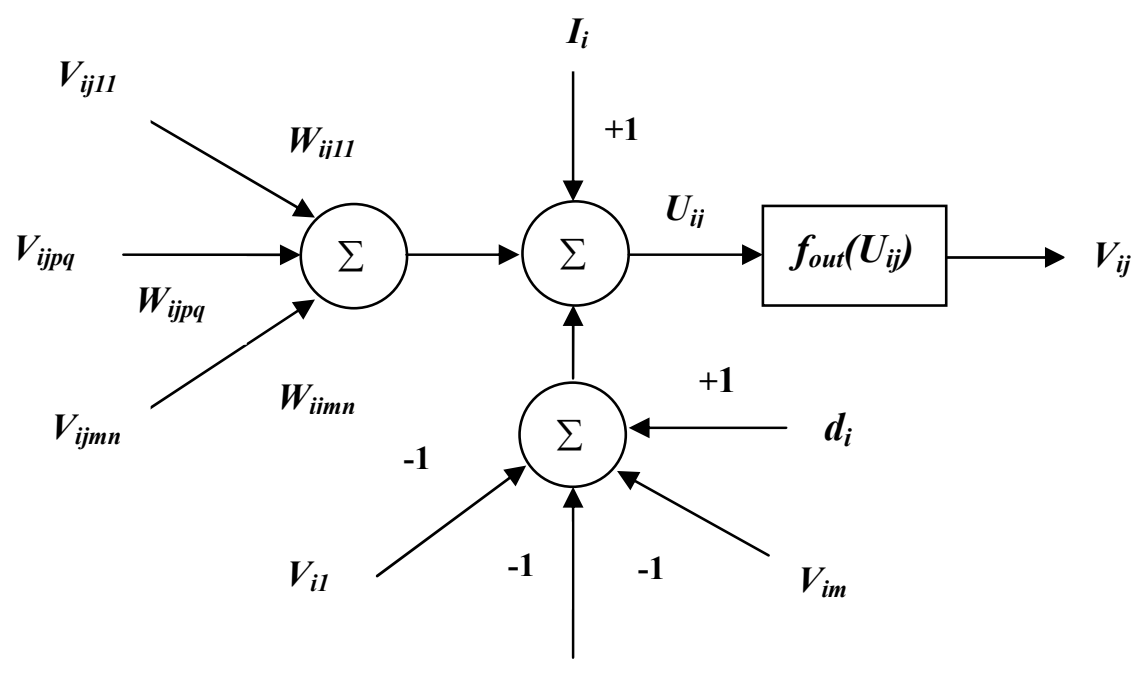

$V_{i j}$

Figure 3. A 2-D Hopfield network for the channel-assignment problem

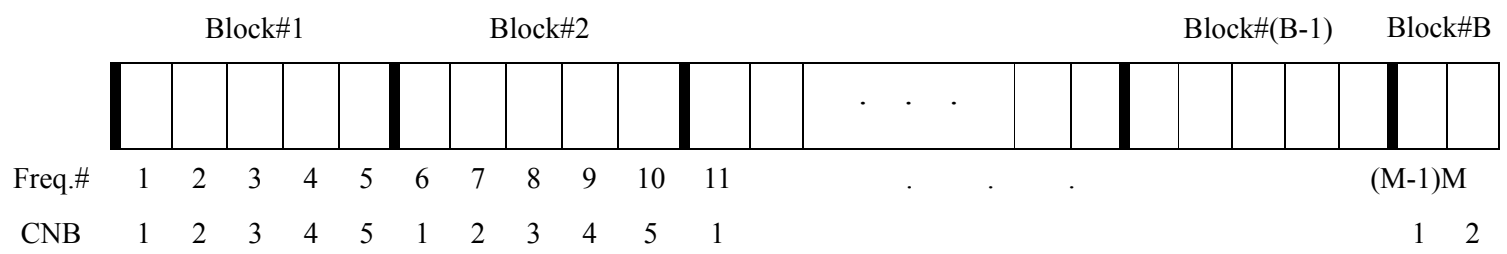

(a)

Freq.\# j $\mathrm{j}+\mathrm{BW}$ $\mathrm{j}+2 \mathrm{BW}$ $\mathrm{j}+3 \mathrm{BW}$
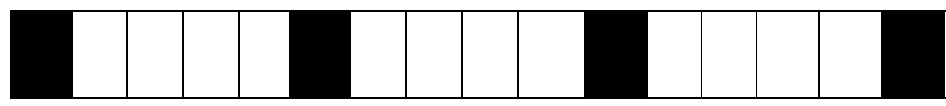

(b)

Figure 4. Structure of frequency domain and initialization: (a) frequency and block structure,

(b) regular interval initialization method

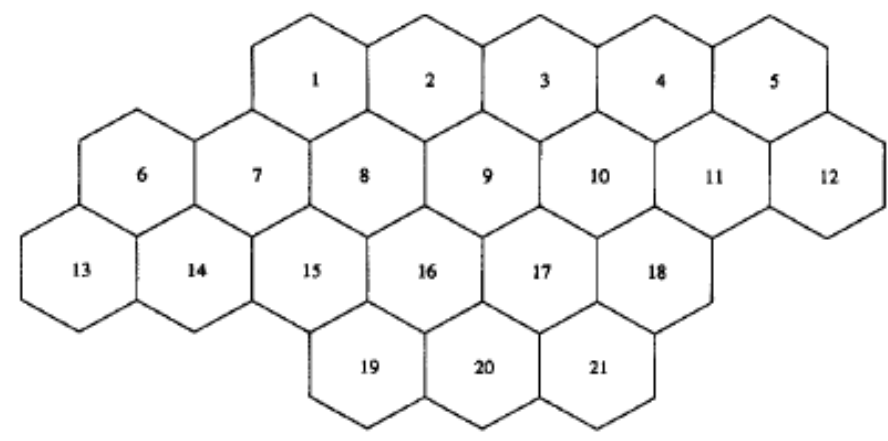

Figure 5. The 21-cell system used in this paper 


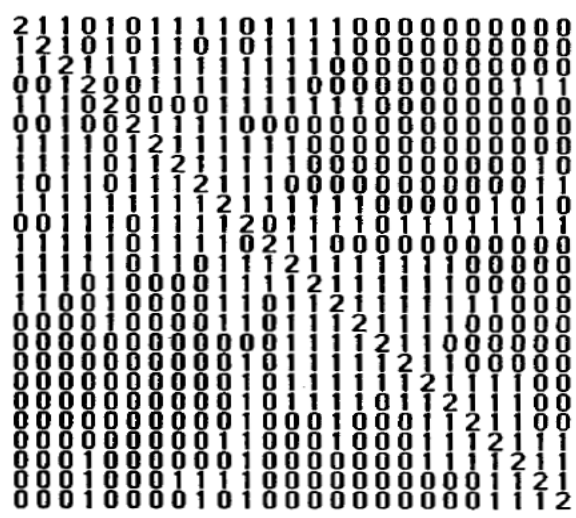

(a)

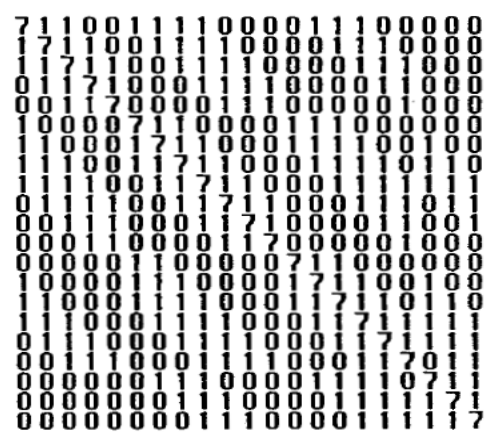

(e)

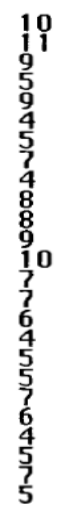

(b)

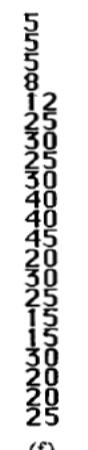

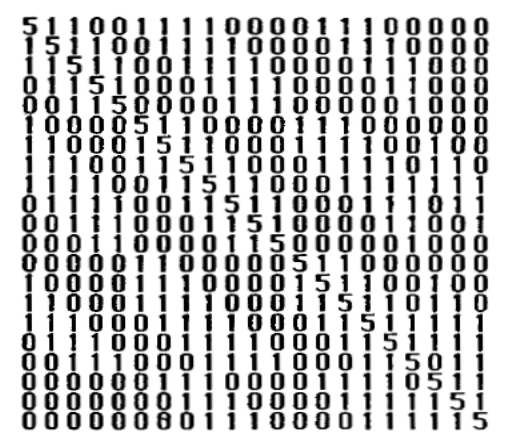

(c)

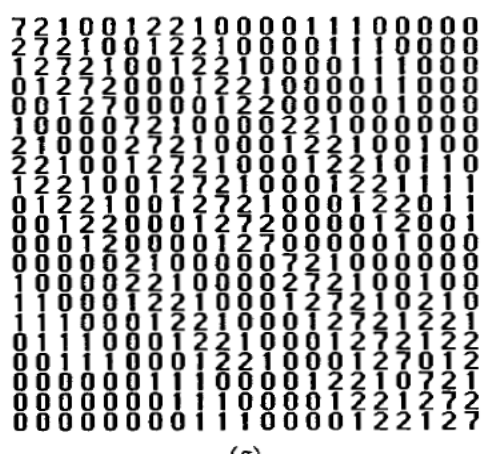

(g)

Figure 6. Compatibility matrices and demand vectors in simulated problems. (a) Compatibility matrix $C_{2}$. (b) Demand vector $D_{2}$. (c) Compatibility matrix $C_{3}$. (d) Demand vector $D_{3}$. (e) Compatibility matrix $C_{4}$.

(f) Demand vector $D_{4}$. (g) Compatibility matrix $C_{5}$

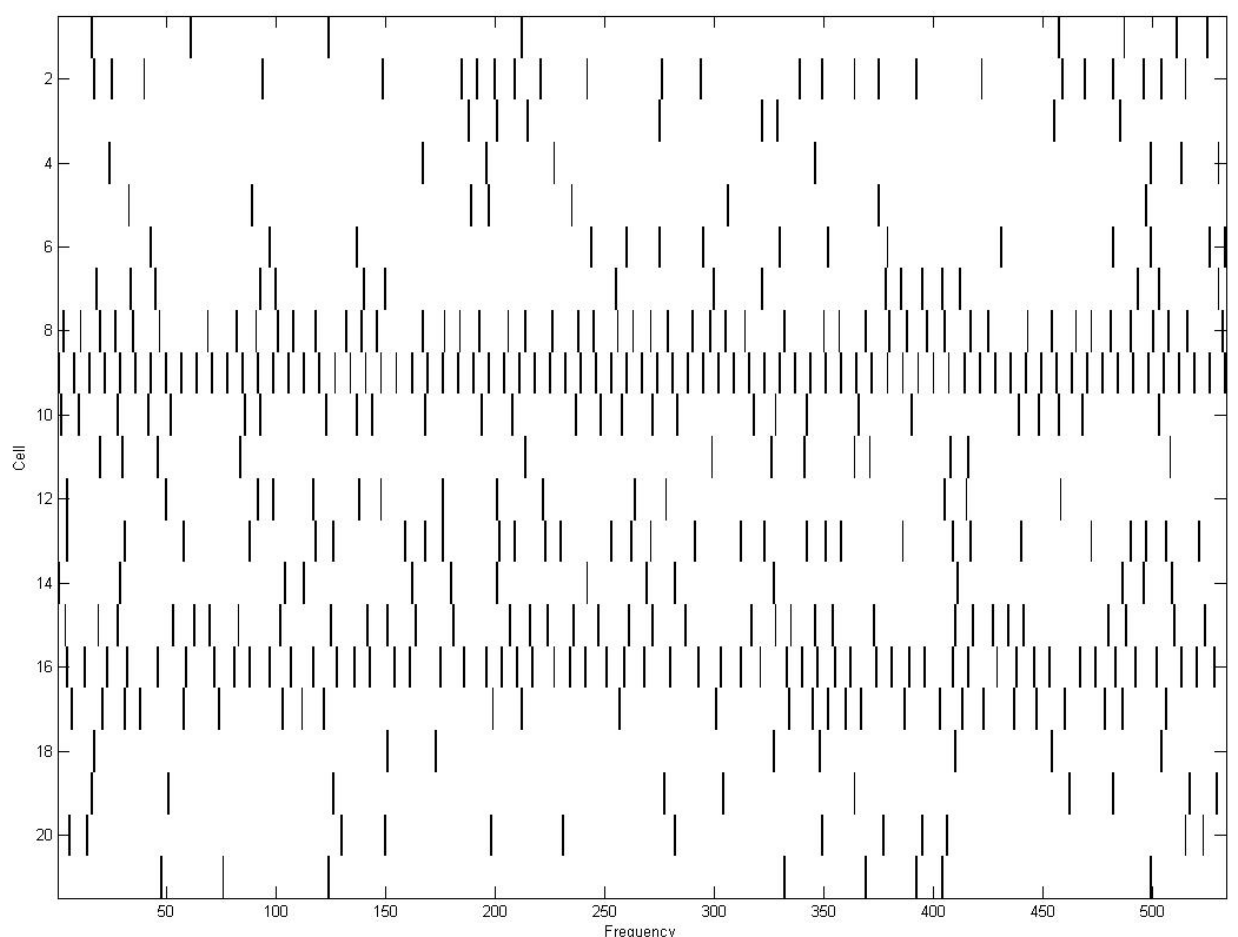

Figure 7. Distribution of the frequency for each cell with 533 frequency in problem \#3. 


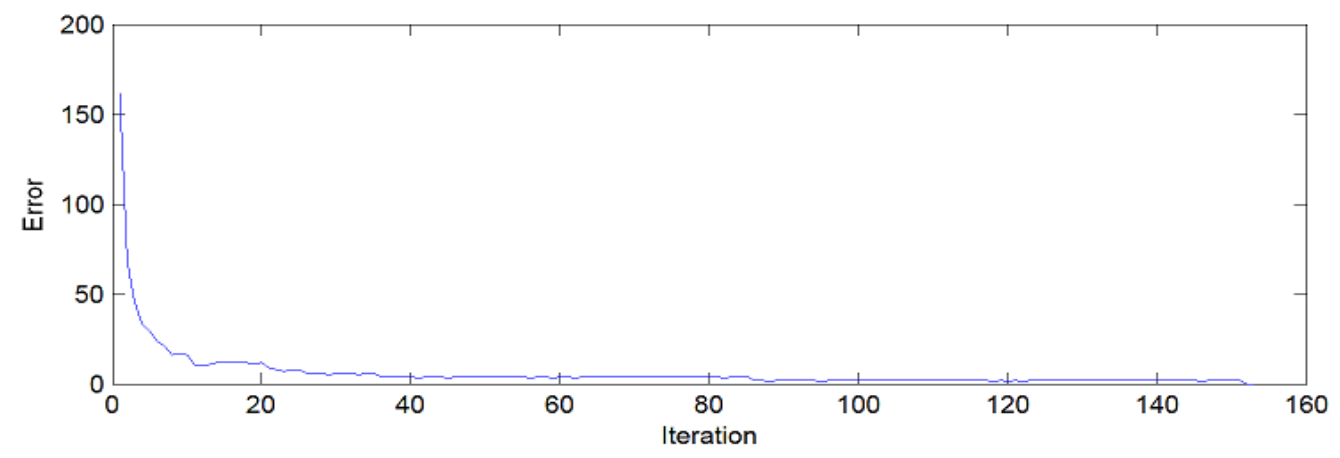

(a)

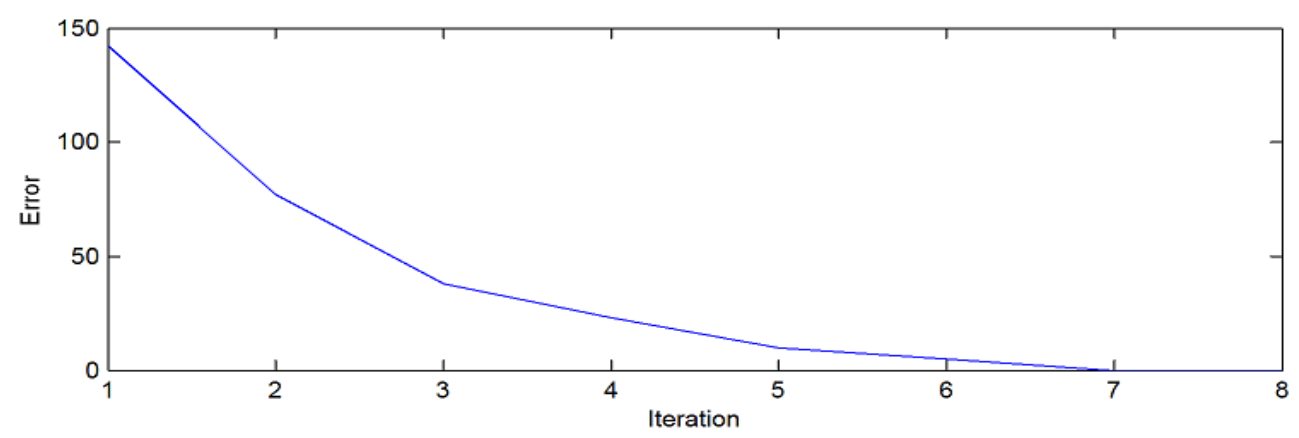

(b)

Figure 8. two typical convergence rate trajectory: (a) Problem \#1 and (b) Problem \#2 